\section{DIN EN ISO 17511}

G. Schumann

Hannover, Deutschland

\section{Englischer Begriff ISO 17511}

Beschreibung Die internationale Norm „In-vitro-Diagnostika - Messung von Größen in Proben biologischen Ursprungs - Metrologische Rückführbarkeit von Werten, die Kalibriermaterialien und Kontrollmaterialien zugeordnet sind" enthält die Anforderungen für die metrologische Rückführung von Messwerten. Durch eine ununterbrochene Kette von Kalibrierungen sollen Messwerte, die mit Routineverfahren ermittelt werden, auf die im Referenzsystem erzielbare Genauigkeit rückgeführt werden. Unter den Bedingungen eines optimalen Referenzsystems endet die metrologische Rückführbarkeitskette bei den international festgelegten Einheiten (engl.: SI units).
Die Norm in der Fassung von 2003 wird im Technischen Komitee 212 (TC212) von ISO grundlegend überarbeitet. Das metrologische Niveau der Rückführung einer Messgröße wird in mehreren Kategorien abgehandelt. In Abhängigkeit von der Verfügbarkeit eines Referenzmaterials und/oder einer Referenzprozedur gibt es dazu Diagramme mit Darstellung der Kalibrierung und der metrologischen Rückführung. Die Norm ISO 18153 ( $\triangleright$ DIN EN ISO 18153) mit der Beschreibung der metrologische Rückführung von Verfahren zur Bestimmung der katalytischen Konzentration von Enzymen wird in die revidierte ISO 17511 eingearbeitet. Die revidierte Norm ISO 17511 wird voraussichtlich 2018 verabschiedet sein.

\section{Literatur}

DIN EN ISO 17511 (2003) In-vitro-Diagnostika - Messung von Größen in Proben biologischen Ursprungs - Metrologische Rückführbarkeit von Werten, die Kalibriermaterialien und Kontrollmaterialien zugeordnet sind. Beuth-Verlag, Berlin 